\title{
Prognostic Impact of Prolonged Cross-clamping Time in Coronary Artery Bypass Grafting
}

Short title: Cross-clamping time in CABG

Vito G. Ruggieri, ${ }^{1}$ Karl Bounader,${ }^{1}$ Jean Philippe Verhoye,${ }^{1}$ Francesco Onorati, ${ }^{2}$ Antonino S. Rubino, ${ }^{3}$ Giuseppe Gatti, ${ }^{4}$ Tuomas Tauriainen, ${ }^{5}$ Marisa De Feo, ${ }^{6}$ Daniel Reichart, ${ }^{7}$ Magnus Dalén, ${ }^{8}$ Peter Svenarud, ${ }^{8}$ Giuseppe Faggian, ${ }^{2}$ Giuseppe Santarpino, ${ }^{9}$ Daniele Maselli, ${ }^{10}$ Riccardo Gherli, ${ }^{11}$ Giovanni Mariscalco, ${ }^{12}$ Antonio Salsano, ${ }^{13}$ Francesco Nicolini, ${ }^{14}$ Tiziano Gherli, ${ }^{14}$ Matteo Saccocci, ${ }^{15}$ Juhani KE Airaksinen, ${ }^{16}$ Sidney Chocron, ${ }^{17}$ Andrea Perrotti, ${ }^{17}$ and Fausto Biancari ${ }^{5,16,18}$

${ }^{1}$ Division of Cardiothoracic and Vascular Surgery, Pontchaillou University Hospital, Rennes, France;

${ }^{2}$ Division of Cardiovascular Surgery, Verona University Hospital, Verona, Italy;

${ }^{3}$ Centro Clinico-Diagnostico “G.B. Morgagni”, Centro Cuore, Pedara, Italy;

${ }^{4}$ Division of Cardiac Surgery, Ospedali Riuniti, Trieste, Italy;

${ }^{5}$ Department of Surgery, Oulu University Hospital and University of Oulu, Oulu, Finland;

${ }^{6}$ Division of Cardiac Surgery, Department of Cardiothoracic Sciences, Second University of Naples, Naples, Italy;

${ }^{7}$ Hamburg University Heart Center, Hamburg, Germany;

${ }^{8}$ Department of Molecular Medicine and Surgery, Department of Cardiothoracic Surgery and Anesthesiology, Karolinska Institutet, Karolinska University Hospital, Stockholm, Sweden;

${ }^{9}$ Cardiovascular Center, Paracelsus Medical University, Nuremberg, Germany;

${ }^{10}$ Department of Cardiac Surgery, St. Anna Hospital, Catanzaro, Italy;

${ }^{11}$ Department of Cardiovascular Sciences, Cardiac Surgery Unit, S. Camillo-Forlanini Hospital, Rome, Italy;

${ }^{12}$ Department of Cardiovascular Sciences, Clinical Sciences Wing, University of Leicester, Glenfield Hospital, Leicester, UK;

${ }^{13}$ Division of Cardiac Surgery, University of Genoa, Genoa, Italy;

${ }^{14}$ Division of Cardiac Surgery, University of Parma, Parma, Italy;

${ }^{15}$ Department of Cardiac Surgery, Centro Cardiologico-Fondazione Monzino IRCCS, University of Milan, Italy;

${ }^{16}$ Heart Center, Turku University Hospital, and University of Turku, Turku, Finland;

${ }^{17}$ Department of Thoracic and Cardio-Vascular Surgery, University Hospital Jean Minjoz, Besançon, France;

${ }^{18}$ Department of Surgery, University of Turku, Turku, Finland.

For correspondence:

Prof. Fausto Biancari,

Heart Center,

Turku University Hospital,

P.O. Box 5000, FI-90014 Oulu, Finland;

Tel.: +358 407333973 ;

E-mail: faustobiancari@yahoo.it 


\section{Abstract}

Background: The prognostic impact of cross-clamping time (XCT) in patients undergoing isolated coronary artery bypass grafting (CABG) has not been thoroughly investigated.

Material and methods: 2957 patients who underwent on-pump isolated CABG from the prospective multicenter E-CABG study were the subjects of this analysis.

Results: The mean XCT in this series was $58 \pm 25$ min. XCT was $>60$ min in 1134 patients $(38.3 \%$ ), $>75 \mathrm{~min}$ in 619 patients (20.9\%) and >90 $\mathrm{min}$ in 296 patients (10.0\%). Multivariate analysis showed that XCT was an independent predictor of 30-day mortality $(\mathrm{p}<0.0001$, OR $1.027,95 \%$ CI 1.015 1.039) along with age $(\mathrm{p}<0.0001)$, female gender $(\mathrm{p}=0.001)$, pulmonary disease $(\mathrm{p}=0.001)$, poor mobility $(\mathrm{p}=0.002)$, urgency status $(\mathrm{p}=0.007)$, critical preoperative status $(\mathrm{p}=0.002)$ and participating centers $(\mathrm{p}=0.015)$. Adjusted risk of 30-day mortality was highest for XCT $>75 \min (2.9 \%$ vs. $1.7 \%$, $\mathrm{p}=0.002$, OR $3.479,95 \%$ CI $1.609-7.520$ ). Analysis of 428 propensity score matched pairs showed that XCT $>75$ min was associated with significantly increased risk of early mortality, prolonged use of inotropes, postoperative use of intra-aortic balloon pump, use of extracorporeal membrane oxygenation, atrial fibrillation, prolonged stay in the intensive care unit and of composite major adverse events.

Conclusions: Isolated CABG is currently performed with prolonged XCT in a significant number of patients and this seems to be a determinant of poor early outcome.

Key words: Coronary artery bypass; CABG; cardiac surgery; cross-clamping; cross clamp; myocardial ischemia. 


\section{Introduction}

Perioperative myocardial damage is considered a main determinant of outcome in patients undergoing cardiac surgery [1-3]. Prolonged aortic cross-clamping time (XCT) is associated with release of myocardial enzymes as a response to significant myocardial ischemia [1,2]. Previous studies suggested that XCT is an independent predictor of a number of adverse events [4-7]. The negative effect of prolonged XCT is likely exacerbated by prolonged cardiopulmonary bypass (CPB) as these parameters strongly correlate each other [4]. However, the evidence of its negative prognostic impact is based on a few studies of retrospective nature including patients undergoing different types of procedures [4-7]. The impact of prolonged XCT has not been investigated in patients undergoing isolated coronary artery bypass grafting $(\mathrm{CABG})$ and there is scarce data on its safe time limit. The present study sought to investigate these issues in patients who underwent $\mathrm{CABG}$ from a prospective, multicenter European registry.

\section{Methods}

\section{Patient population}

The E-CABG registry is a prospective, multicenter study enrolling patients undergoing isolated CABG at 16 European centers of cardiac surgery (Besançon, France; Catanzaro, Italy; Genoa, Italy; Hamburg, Germany; Milan, Italy; Nuremberg, Germany; Naples, Italy; Oulu, Finland; Parma, Italy; Pedara, Italy; Rennes, France; Rome, Italy; Stockholm, Sweden; Trieste, Italy; Verona, Italy). Twelve centres are university hospitals, two centres are central hospitals and two centres are private hospitals with agreements with the regional health authorities. The E-CABG study is registered in Clinicaltrials.gov (Identifier: NCT02319083) and its detailed protocol along with definition criteria are reported elsewhere [8]. The Institutional or Regional Review Board of all the participating centers approved this study. The informed consent was collected in Institutions where the internal Institutional Review Board required it, otherwise it was waived. This study was not financially supported.

Only patients who underwent on-pump CABG with arrest were considered for the present analysis. 
Patients who underwent off-pump CABG, on-pump CABG with arrest after conversion of an offpump procedure and those who underwent heart-beating CABG on pump were excluded from this study. Details on the methods of myocardial protection (cardioplegia type, topical cooling and cardioplegia temperature are summarized in Table 1.

\section{Outcomes}

The primary outcome of this study was in-hospital/30-day mortality. Secondary end-points were prolonged inotropic support, postoperative use of intra-aortic balloon pump and extracorporeal membrane oxygenation, sternal wound infection, acute kidney injury, renal replacement therapy, delirium, stroke, resternotomy for bleeding, percutaneous coronary intervention, atrial fibrillation, intensive care unit stay as well as the composite outcome E-CABG complication grades 3-4.

The severity of postoperative renal dysfunction was defined according to the AKIN criteria [9]. The ECABG complication grades 3-4 was employed as a composite measure of adverse outcome and includes transfusion of $>10$ units of red blood cells, renal failure required dialysis, mediastinitis, stroke, early repeat revascularization, reoperation for hemodynamic instability, ventricular fibrillation/asystole, surgery for gastrointestinal complications, need of extracorporeal membrane oxygenation and/or in-hospital/30-day death [8].

\section{Statistical Analysis}

Statistical analysis was performed using the SPSS v. 23.0 statistical software (IBM Corporation, 1 New Orchard Road Armonk, New York, USA). No attempt to replace missing values was made.

Fisher's exact test, Chi-square test, Mann-Whitney U-test, Kruskal-Wallis test, t-test for paired samples for continuous variables and McNemar test were used for univariate analysis. Linear regression was employed to identify risk factors associated with prolonged XCT including the following covariates: participating center, diabetes, dialysis, prior percutaneous coronary intervention, prior cardiac surgery, Syntax score, number of diseased vessels, use of bilateral internal mammary 
artery grafting, use of radial artery graft and number of distal anastomoses. Logistic regression was used to adjust the effect of XCT on the early outcome for the following clinical and operative covariates: age, gender, estimated glomerular filtration rate, stroke, poor mobility, extracardiac arteriopathy, diabetes, pulmonary disease, atrial fibrillation, left ventricular ejection fraction, recent myocardial infarction, urgency of the operation, critical preoperative status, prior percutaneous coronary intervention, prior cardiac surgery, Syntax score, left main stenosis, number of distal anastomoses, use of bilateral internal mammary artery grafts, use of radial artery graft, blood vs. crystalloid cardioplegia, temperature of cardioplegia, route of cardioplegia administration, topical cooling and participating centres. These covariates were used in all multivariate analyses using either enter or backward selection methods. The reliability of the regression models was assessed with the Cstatistic and the Hosmer-Lemeshow's test. The Youden's test was used to identify the best cutoff of XCT in predicting 30-day mortality. Since the Youden's index does not provide a multiple covariateadjusted estimate of the cut-off value, adjusted odds ratios of increasing cut-off values of XCT were estimated including the above-mentioned covariates. The cut-off value with the highest adjusted odds ratio for early mortality was employed for further analyses. A propensity score was estimated using this cut-off value of XCT as the dependent variable and the above listed variables as covariates. Oneto-one propensity score matching was performed employing a caliper of 0.2 of the standard deviation of the logit of the propensity score. To evaluate the balance between the matched groups, the t-test for paired samples for continuous variables, the McNemar test for dichotomous variables and the analysis of the standardized differences after matching have been used. Standardized differences less than $10 \%$ were considered as acceptable imbalance. $\mathrm{P}<0.05$ was considered statistically significant.

\section{Results}

\section{Incidence and predictors of prolonged XCT}

Out of 3788 consecutive patients operated on from January to December 2015 and enrolled in the ECABG registry, 2962 patients underwent on-pump CABG with arrest. In the present study, 2957 
patients $(99.8 \%)$ with complete data on XCT were the subjects of this analysis (Tab. 1). The mean $\mathrm{XCT}$ in this series was $58 \pm 25 \min$ (median 53 , interquartile range $31 \mathrm{~min}$ ). The mean $\mathrm{CPB}$ time was $84 \pm 34 \mathrm{~min}$ (median 79, interquartile range $40 \mathrm{~min}$ ) and was significantly correlated with XCT (rho: $0.863, \mathrm{p}<0.0001)$.

$\mathrm{XCT}$ was $>45 \mathrm{~min}$ in 1879 patients $(63.5 \%),>60 \mathrm{~min}$ in 1134 patients $(38.3 \%),>75 \mathrm{~min}$ in 619 patients $(20.9 \%)$ and $>90 \mathrm{~min}$ in 296 patients $(10.0 \%)$.

XCT correlated significantly with Syntax score (rho: 0.194, p<0.0001), number of diseased vessels (rho: 0.316, p<0.0001) and number of distal anastomoses (rho: 0.585, p<0.0001). XCT was significantly prolonged in patients undergoing CABG using bilateral internal mammary artery grafts (65 \pm 25 vs. $53 \pm 23 \mathrm{~min}, \mathrm{p}<0.0001)$ and radial artery graft $(69 \pm 21$ vs. $57 \pm 25 \mathrm{~min}, \mathrm{p}<0.0001) . \mathrm{XCT}$ varied significantly between centers from $38 \pm 10 \mathrm{~min}$ to $90 \pm 21 \mathrm{~min}(\mathrm{p}<0.0001)$. Similarly, also Syntax score $(\mathrm{p}<0.0001)$, number of diseased vessels $(\mathrm{p}<0.0001)$ and number of distal anastomoses $(p<0.0001)$ differed significantly between centers.

Linear regression showed that participating centers $(\mathrm{p}=0.034)$, Syntax score $(\mathrm{p}=0.001)$, number of diseased vessels $(\mathrm{p}<0.0001)$, number of distal anastomoses $(\mathrm{p}<0.0001)$, bilateral internal mammary artery grafting $(\mathrm{p}<0.0001)$ and radial artery grafting $(\mathrm{p}<0.0001)$ were independent predictors of prolonged XCT.

\section{Prognostic impact of prolonged XCT}

In this series, XCT was associated with an increased risk of 30-day mortality $(\mathrm{p}=0.014$, area under the ROC curve $0.595,95 \%$ CI $0.522-0.668)$.

Multivariate analysis (Hosmer-Lemeshow's test: $\mathrm{p}=0.466$, area under the ROC curve $0.901,95 \% \mathrm{CI}$ 0.865-0.936) showed that XCT was an independent predictor of 30-day mortality $(\mathrm{p}<0.0001$, OR 1.027, 95\% CI 1.015-1.039) along with age $(\mathrm{p}<0.0001)$, female gender $(\mathrm{p}=0.001)$, pulmonary disease $(\mathrm{p}=0.001)$, poor mobility $(\mathrm{p}=0.002)$, urgency status $(\mathrm{p}=0.007)$, critical preoperative status $(\mathrm{p}=0.002)$ and participating centers $(\mathrm{p}=0.015)$. When logarithmically transformed, XCT was still an independent 
predictor of early mortality ( $\mathrm{p}<0.0001$, OR $5.535,95 \%$ CI $2.345-13.065)$. The type of cardioplegia, the routes of its administration and its temperate were not predictive of early mortality.

Adjusted analysis showed that the risk of 30-day mortality increased significantly along with increasing quartiles of XCT (Fig. 1). Youden's test showed that a cut-off value of 53 minutes was associated with a significantly increased risk of 30 -day mortality ( $2.6 \%$ vs. $1.3 \%$, unadjusted $\mathrm{p}=0.009)$. XCT longer than 53 min adjusted for the above-mentioned covariates was an independent predictor of 30-day mortality ( $\mathrm{p}=0.009$, OR 2.791, 95\%CI 1.290-6.038).

Since the Youden's index does not provide a covariate-adjusted estimate of the cut-off value, adjusted odds ratios were estimated for increasing XCT of 5 min (Fig. 2). This adjusted analysis showed that early mortality was highest when XCT $>75 \min (2.9 \%$ vs. $1.7 \%$, p=0.002, OR 3.479, 95\%CI 1.6097.520). Therefore, this value was employed to assess the risk of other adverse events with prolonged XCT (Tab. 2). This analysis showed that XCT $>75$ min was an independent predictor of prolonged treatment with inotropes, use of intra-aortic balloon pump and extracorporeal membrane oxygenation, need of postoperative percutaneous coronary intervention, acute kidney injury, intensive care unit stay $>2$ days as well as of combined E-CABG complication grades 3-4 (Tab. 2).

\section{Propensity score matching analysis}

Logistic regression (Hosmer-Lemeshow's test: $\mathrm{p}=0.323$ ) provided a propensity score with an area under the ROC curve of $0.872(95 \% \mathrm{CI} 0.856-0.887)$ and this was used for matching using a caliper width of 0.05 . Propensity score matching resulted in 428 pairs with similar baseline and operative characteristics as shown by standardized differences $<10 \%$ in all covariates (Tab. 1). Analysis of propensity score matched pairs showed that XCT $>75$ min was associated with significantly increased risk of in-hospital/30-day mortality, prolonged use of inotropes, postoperative use of intra-aortic balloon pump, use of extracorporeal membrane oxygenation, atrial fibrillation, prolonged stay in the intensive care unit and of composite E-CABG complication grades 3-4. 


\section{Discussion}

The present study showed that isolated CABG is currently performed with prolonged period myocardial ischemia in a significant proportion of patients and that XCT is an independent predictor of early adverse events. These results comply with those of a few available studies focusing on this issue [4-7].

This study allowed us to identify a threshold of XCT in patients undergoing isolated CABG (Fig. 2). Although unadjusted analysis showed that a XCT >53 min was associated with an increased risk of early death, adjusted analysis showed that such a risk was highest when XCT was longer than $>75$ min (Fig. 2). Propensity score matching confirmed that XCT $>75$ min was associated with significantly increased risk of in-hospital/30-day mortality and provided evidence of its negative prognostic impact on the risk of prolonged use of inotropes, postoperative use of intra-aortic balloon pump, use of extracorporeal membrane oxygenation, atrial fibrillation, prolonged stay in the intensive care unit and of composite E-CABG complication grades 3-4. These observations confirmed the intuitively increased risk of cardiac-related complications secondary to prolonged intraoperative myocardial ischemia.

The present findings are of clinical importance as a previous study from a general cardiac surgery population identified a cutoff of $150 \mathrm{~min}$ as a threshold of myocardial ischemia [4]. Two other studies evaluated different durations of XCT without providing any prognostic threshold value for XCT [5,7]. The most recent study included patients who underwent isolated aortic valve replacement and confirmed that the risk of operative mortality was significantly increased in patients in whom XCT was longer than 150 min (adjusted OR 2.68, 95\%CI 1.66-4.32). A study by Al-Sarraf et al. [5] showed that the risk of in-hospital mortality was increased when XCT > 60 min and it was highest when XCT $>90$ min. Based on these findings and the results of the present study, we suspect that the threshold of XCT could be much shorter in patients undergoing isolated CABG compared with valve surgery. In this setting, a shorter duration of myocardial ischemia seems to be of prognostic importance and 
adequate myocardial protection could be a critical issue in patients with severe coronary artery disease. Indeed, methods of myocardial protection might have influenced the outcome of these patients and therefore blood vs. crystalloid cardioplegia, temperature of cardioplegia, route of its administration, topical cooling as well as participating centres were included in all regression analyses. The rather small size of this study and the myriad of methods of myocardial protection employed in these patients prevented a subgroup analysis of the prognostic impact of different cardioplegias, their temperatures and the routes of administration as well as of the use of topical cooling.

An interesting finding of this study was that XCT varied significantly between centers. Individual surgeon's skills and experience is probably accountable for this variability. Recent studies demonstrated that supervised residents may perform CABG with results similar to attending surgeons [10-13). Beside the fact that analysis of results of surgeons in training might be biased by a number of educational factors [14], the severity of coronary disease and the number of distal anastomoses performed by resident and attending surgeons were taken in to account $[10,11]$. Furthermore, there is data suggesting that the operative risk is higher in patients operated on by attending surgeons [12]. It is clear that residents accomplish CABG with longer XCT [11,12]. These observations suggest that $\mathrm{CABG}$ is often a technically demanding procedure which requires a meticulous technique and the ability to accomplish surgical revascularization within a short period of myocardial ischemia. In the present study, multivariate analysis showed that Syntax score $(\mathrm{p}=0.001)$, number of diseased vessels $(\mathrm{p}<0.0001)$, number of distal anastomoses $(\mathrm{p}<0.0001)$, bilateral internal mammary artery grafting $(\mathrm{p}<0.0001)$ and radial artery grafting $(\mathrm{p}<0.0001)$ were among the independent predictors of prolonged $\mathrm{XCT}$. These findings suggest that expertise in coronary surgery is required particularly in patients with multivessel disease and when the use of multiple arterial grafts is anticipated. These factors should be taken into account when planning surgical training in order to not jeopardize the technical success of the procedure and the outcome of patients. 
The strength of this study resides in its prospective nature and the availability of a number of covariates for stratification of the severity of coronary artery disease as well as details on myocardial protection. Still several limitations might affect this analysis and deserve to be acknowledged. First, this registry collects data from institutions with different referral pathways, revascularization strategies and myocardial protection methods. These covariates were included in all regression models along with the participating centers, but adjustment methods might still be suboptimal. Furthermore, the relatively limited size of this series prevented sensitivity analyses. Second, although the prognostic effects of XCT was adjusted for a large number of baseline and operative variables, it is possible that prolonged myocardial ischemia time was related to technical or anatomical factors which cannot be taken into consideration in the present analysis. Third, identification of a threshold of XCT is complicated by the presence of several technical factors and comorbidities which may affect both XCT and postoperative outcome. Therefore, the cut-off value identified by the Youden's test was not employed for further analysis. Instead a cut-off of XCT with the highest adjusted risk estimate of early mortality was considered for evaluation of major adverse events.

In conclusion, this study showed that isolated CABG is currently performed with prolonged XCT in a significant number of patients. Multivariate analysis showed that prolonged XCT seems to be a determinant of poor early outcome. Therefore, strategies to reduce XCT and methods to improve myocardial protection when prolonged arrest is expected may decrease the risk of early adverse events after CABG. Further studies from larger series are needed to verify these findings.

\section{Acknowledgment}

This study was not financially supported.

\section{Conflict of interest}

None. 


\section{References}

1. Onorati F, De Feo M, Mastroroberto P, et al. Determinant and prognosis of myocardial damage after coronary artery bypass grafting. Ann Thorac Surg. 2005;79:837-845.

2. Croal BL, Hillis GS, Gibson PH, et al. Relationship between postoperative cardiac troponin I levels and outcome of cardiac surgery. Circulation. 2006;114:1468-75.

3. Eigel P, van Ingen G, Wagenpfeil S. Predictive value of perioperative cardiac troponin I for adverse outcome in coronary artery bypass surgery. Eur J Cardiothorac Surg. 2001;20:544-9.

4. Nissinen J, Biancari F, Wistbacka JO, et al. Safe time limits of aortic cross-clamping and cardiopulmonary bypass in adult cardiac surgery. Perfusion. 2009;24:297-305.

5. Al-Sarraf N, Thalib L, Hughes A, et al. Cross-clamp time is an independent predictor of mortality and morbidity in low-and high-risk cardiac patients. Int J Surg. 2011;9:104-9.

6. Doenst T, Borger MA, Weisel RD, Yau TM, Maganti M, Rao V. Relation between aortic crossclamp time and mortality: not as straightforward as expected. Eur J Cardiothorac Surg. 2008;33:660-5.

7. Iino $\mathrm{K}$, Miyata $\mathrm{H}$, Motomura $\mathrm{N}$, et al. Prolonged cross-clamping during aortic valve replacement is an independent predictor of postoperative morbidity and mortality: analysis of the Japan Cardiovascular Surgery Database. Ann Thorac Surg. 2017;103:602-609.

8. Biancari F, Ruggieri VG, Perrotti A, et al. European multicenter study on coronary artery bypass Grafting (E-CABG registry): study protocol for a prospective clinical registry and proposal of classification of postoperative complications. J Cardiothorac Surg. 2015;10:90.

9. Mehta RL, Kellum JA, Shah SV, et al. Acute Kidney Injury Network: report of an initiative to improve outcomes in acute kidney injury. Crit Care. 2007;11:R31.

10. Almassi GH, Carr BM, Bishawi M, et al; Veterans Affairs \#517 Randomized On/Off Bypass (ROOBY) Study Group. Resident versus attending surgeon graft patency and clinical outcomes in on- versus off-pump coronary artery bypass surgery. J Thorac Cardiovasc Surg. 2015;150:142835. 
11. Bakaeen FG, Sethi G, Wagner TH, et al. Coronary artery bypass graft patency: residents versus attending surgeons. Ann Thorac Surg. 2012;94:482-8.

12. Yount KW, Yarboro LT, Narahari AK, et al. Outcomes of trainees performing coronary artery bypass grafting: does resident experience matter? Ann Thorac Surg. 2017;103:975-981.

13. Bakaeen FG, Dhaliwal AS, Chu D, et al. Does the level of experience of residents affect outcomes of coronary artery bypass surgery? Ann Thorac Surg. 2009;87:1127-33

14. Mokadam NA. The evidence is suspect. J Thorac Cardiovasc Surg. 2016;151:1216. 


\section{Legend to figures}

Figure 1. Thirty-day mortality rates per quartiles of aortic cross-clamping time. Adjusted odds ratios (OR) and $95 \%$ confidence intervals $(95 \% \mathrm{CI})$ are reported for each increasing quartile.

Figure 2. Adjusted odds ratios and $95 \%$ confidence intervals $(95 \% \mathrm{CI})$ for early mortality according to different cutoff values of aortic cross-clamping time. 
Table 1. Baseline characteristics of the study cohorts.

\begin{tabular}{|c|c|c|c|c|c|c|c|c|}
\hline & Overall series & & & & Propensity & matched pairs & & \\
\hline Baseline variables & $\begin{array}{c}X C T \leq 75 \mathrm{~min} \\
(n=2338)\end{array}$ & $\begin{array}{c}X C T>75 \min \\
(n=619)\end{array}$ & p-value & $\begin{array}{l}\text { Standardized } \\
\text { difference (\%) }\end{array}$ & $\begin{array}{c}X C T \leq 75 \mathrm{~min} \\
(n=428)\end{array}$ & $\begin{array}{c}X C T>75 \min \\
(n=428)\end{array}$ & p-value & $\begin{array}{l}\text { Standardized } \\
\text { difference (\%) }\end{array}$ \\
\hline Age (years) & $67.0 \pm 9.3$ & $66.8 \pm 8.9$ & 0.436 & 3 & $66.6 \pm 9.0$ & $67.2 \pm 8.8$ & 0.342 & 7 \\
\hline Females & 387 (16.6) & 87 (14.1) & 0.132 & 7 & 68 (15.9) & $66(15.4)$ & 0.851 & 1 \\
\hline $\mathrm{eGFR}\left(\mathrm{mL} / \mathrm{min} / 1.73 \mathrm{~m}^{2}\right)$ & $82 \pm 26$ & $81 \pm 23$ & 0.721 & 4 & $82 \pm 23$ & $80 \pm 23$ & 0.634 & 5 \\
\hline Stroke & $67(2.9)$ & $24(3.9)$ & 0.195 & 6 & $15(3.5)$ & $16(3.7)$ & 0.855 & 1 \\
\hline Poor mobility & $56(2.4)$ & $7(1.1)$ & 0.053 & 10 & $4(0.9)$ & $6(1.4)$ & 0.752 & 5 \\
\hline Extracardiac arteriopathy & $471(20.1)$ & $120(19.4)$ & 0.674 & 2 & $93(21.7)$ & $89(20.8)$ & 0.738 & 2 \\
\hline Diabetes & $725(31.0)$ & $200(32.3)$ & 0.539 & 3 & $135(31.5)$ & $129(30.1)$ & 0.657 & 3 \\
\hline Pulmonary disease & $197(8.4)$ & $46(7.4)$ & 0.423 & 4 & $33(7.7)$ & $34(7.9)$ & 0.899 & 1 \\
\hline Atrial fibrillation & $154(6.6)$ & $51(8.3)$ & 0.147 & 6 & $34(7.9)$ & $37(8.6)$ & 0.710 & 3 \\
\hline Left ventricular ejection fraction $\leq 50 \%$ & $634(27.1)$ & $162(26.2)$ & 0.621 & 2 & $112(26.2)$ & $109(25.5)$ & 0.815 & 1 \\
\hline Recent myocardial infarction & $702(30.0)$ & $198(32.0)$ & 0.346 & 4 & $122(28.5)$ & $124(29.0)$ & 0.880 & 1 \\
\hline Urgent or emergency oper. & $1075(46.0)$ & $314(50.7)$ & 0.035 & 9 & $220(51.4)$ & $213(49.8)$ & 0.632 & 3 \\
\hline Critical preoperative status & $61(2.6)$ & $39(6.3)$ & $<0.0001$ & 18 & $17(4.0)$ & $21(4.9)$ & 0.507 & 4 \\
\hline Prior cardiac surgery & $15(0.6)$ & $5(0.8)$ & 0.654 & 2 & $4(0.9)$ & $4(0.9)$ & 1.000 & 0 \\
\hline Prior percutaneous coronary interv. & $486(20.8)$ & $123(19.9)$ & 0.626 & 2 & $86(20.1)$ & $86(20.1)$ & 1.000 & 0 \\
\hline Syntax score & $27.2 \pm 10.6$ & $31.0 \pm 9.7$ & $<0.0001$ & 37 & $30.0 \pm 10.4$ & $30.4 \pm 9.7$ & 0.367 & 4 \\
\hline Left main stenosis & $878(37.6)$ & $231(37.3)$ & 0.914 & 1 & $170(39.7)$ & $166(38.8)$ & 0.779 & 2 \\
\hline Bilateral inter. mammary a. grafts & $803(34.3)$ & $312(50.4)$ & $<0.0001$ & 33 & $207(48.4)$ & $211(49.3)$ & 0.784 & 2 \\
\hline Radial a. graft & $30(1.3)$ & $15(2.4)$ & 0.039 & 8 & $6(1.4)$ & $7(1.6)$ & 0.780 & 2 \\
\hline Number of distal anastomoses & $2.6 \pm 0.8$ & $3.5 \pm 0.9$ & $<0.0001$ & 108 & $3.2 \pm 0.8$ & $3.2 \pm 0.7$ & 0.922 & 0 \\
\hline Blood cardioplegia & $1977(84.8)$ & $529(85.5)$ & 0.673 & 3 & $354(82.7)$ & $352(82.2)$ & 0.857 & 1 \\
\hline Topical cooling & $492(21.0)$ & $204(33.0)$ & $<0.0001$ & 27 & $151(35.3)$ & $153(35.7)$ & 0.886 & 1 \\
\hline Cardioplegia temperature $\left({ }^{\circ} \mathrm{C}\right)$ & $21 \pm 15$ & $13 \pm 12$ & $<0.0001$ & 59 & $14 \pm 13$ & $14 \pm 13$ & 0.665 & 2 \\
\hline EuroSCORE II (\%) & $2.4 \pm 3.6$ & $2.7 \pm 4.1$ & 0.015 & 9 & $2.5 \pm 4.5$ & $2.5 \pm 3.9$ & 0.423 & 0 \\
\hline
\end{tabular}


Table 2. Outcomes.

\begin{tabular}{|c|c|c|c|c|c|c|c|}
\hline & \multicolumn{2}{|c|}{ Overall series } & \multirow[b]{2}{*}{ Adjusted p-value } & \multicolumn{4}{|c|}{ Propensity score matched pairs } \\
\hline & $\begin{array}{c}X C T \leq 75 \min \\
(n=2338)\end{array}$ & $\begin{array}{c}X C T>75 \min \\
(n=619)\end{array}$ & & $\begin{array}{c}\text { Adjusted risk } \\
\text { estimate, } 95 \% \text { CI }\end{array}$ & $\begin{array}{c}X C T \leq 75 \min \\
(n=428)\end{array}$ & $\begin{array}{c}X C T>75 \mathrm{~min} \\
(n=428)\end{array}$ & p-value \\
\hline In-hospital/30-day mortality & $39(1.7)$ & $18(2.9)$ & 0.002 & $3.48,1.61-7.52$ & $4(0.9)$ & $15(3.5)$ & 0.019 \\
\hline Prolonged inotropic support & $651(27.8)$ & $241(38.9)$ & $<0.0001$ & $1.86,1.38-2.52$ & $122(28.5)$ & 165 (38.6) & 0.003 \\
\hline Postoperative IABP* & $45(2.0)$ & $24(4.1)$ & 0.024 & $2.22,1.11-4.46$ & $10(2.4)$ & $20(4.9)$ & $<0.0001$ \\
\hline Postoperative ECMO* & $4(0.2)$ & $6(1.0)$ & 0.007 & $27.33,3.48-214.51$ & 0 & $5(1.2)$ & $<0.0001$ \\
\hline Postoperative PCI & $19(0.8)$ & $13(2.1)$ & 0.009 & $3.65,1.38-9.68$ & $3(0.7)$ & $10(2.3)$ & 0.092 \\
\hline Resternotomy for bleeding & $56(2.4)$ & $20(3.2)$ & 0.66 & $0.85,0.42-1.72$ & $9(2.1)$ & $11(2.6)$ & 0.824 \\
\hline Sternal wound infection & $126(5.4)$ & $34(5.5)$ & 0.752 & $0.92,0.56-1.52$ & $20(4.7)$ & $22(5.1)$ & 0.874 \\
\hline Atrial fibrillation & $595(25.6)$ & $174(28.2)$ & 0.361 & $1.13,0.89-1.48$ & $91(21.4)$ & $122(28.6)$ & 0.023 \\
\hline Acute kidney injury & $506(22.0)$ & $179(29.2)$ & 0.006 & $1.451 .11-1.88$ & $99(23.3)$ & $123(29.1)$ & 0.085 \\
\hline Renal replacement therapy & $29(1.3)$ & $13(2.1)$ & 0.17 & $1.84,0.77-4.41$ & $4(0.9)$ & $9(2.1)$ & 0.267 \\
\hline Delirium & $91(3.9)$ & $43(6.9)$ & 0.011 & $2.03,1.17-3.50$ & $17(4.0)$ & $28(6.5)$ & 0.127 \\
\hline Stroke & $32(1.4)$ & $11(1.8)$ & 0.539 & $1.32,0.54-3.24$ & $6(1.4)$ & $7(1.6)$ & 1.000 \\
\hline Intensive care unit stay (days) & $2.7 \pm 3.2$ & $3.1 \pm 7.6$ & 0.031 & $0.35,0.03-0.68$ & $2.4 \pm 2.3$ & $3.1 \pm 8.7$ & $<0.0001$ \\
\hline E-CABG complication grades 3-4 & $133(5.7)$ & $53(8.6)$ & 0.046 & $1.56,1.01-2.41$ & $19(4.4)$ & $36(8.4)$ & 0.025 \\
\hline
\end{tabular}

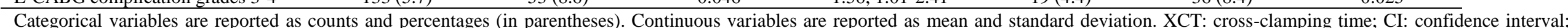

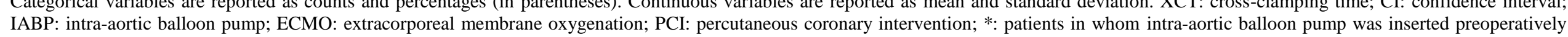
were excluded from the analysis. 
3.5

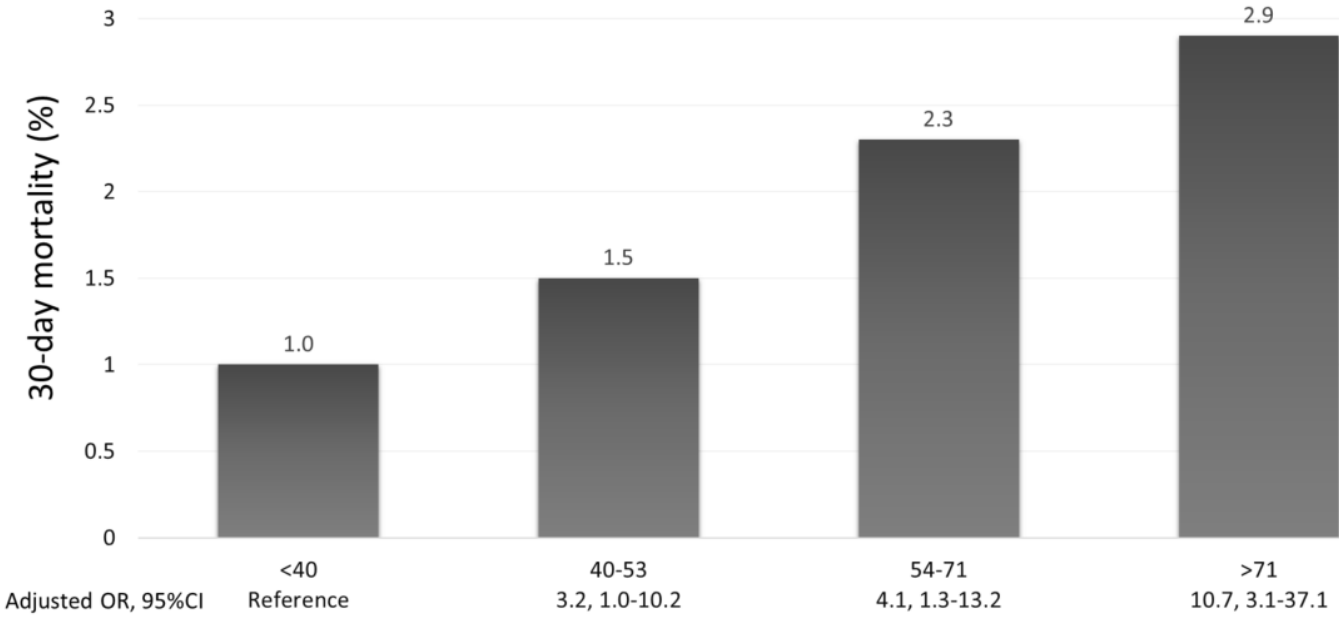

Aortic cross-clamping time (min) 


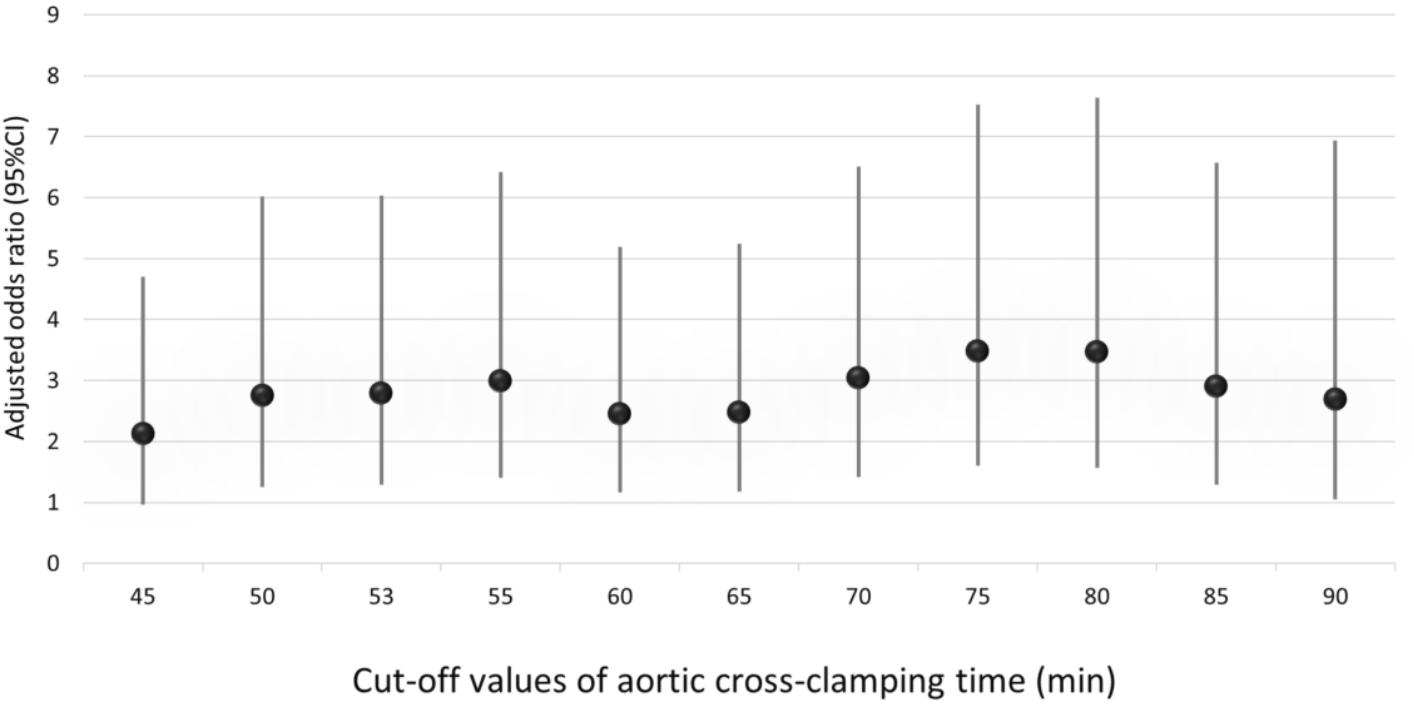

